\title{
Spanners of Complete $k$-Partite Geometric Graphs
}

\author{
Prosenjit Bose* Paz Carmi* Mathieu Couture* Anil Maheshwari* Pat Morin* \\ Michiel Smid*
}

August 3, 2021

\begin{abstract}
We address the following problem: Given a complete $k$-partite geometric graph $K$ whose vertex set is a set of $n$ points in $\mathbb{R}^{d}$, compute a spanner of $K$ that has a "small" stretch factor and "few" edges. We present two algorithms for this problem. The first algorithm computes a $(5+\epsilon)$-spanner of $K$ with $O(n)$ edges in $O(n \log n)$ time. The second algorithm computes a $(3+\epsilon)$-spanner of $K$ with $O(n \log n)$ edges in $O(n \log n)$ time. The latter result is optimal: We show that for any $2 \leq k \leq n-\Theta(\sqrt{n \log n})$, spanners with $O(n \log n)$ edges and stretch factor less than 3 do not exist for all complete $k$-partite geometric graphs.
\end{abstract}

\section{Introduction}

Let $S$ be a set of $n$ points in $\mathbb{R}^{d}$. A geometric graph with vertex set $S$ is an undirected graph $H$ whose edges are line segments $\overline{p q}$ that are weighted by the Euclidean distance $|p q|$ between $p$ and $q$. For any two points $p$ and $q$ in $S$, we denote by $\delta_{H}(p, q)$ the length of a shortest path in $H$ between $p$ and $q$. For a real number $t \geq 1$, a subgraph $G$ of $H$ is said to be a $t$-spanner of $H$, if $\delta_{G}(p, q) \leq t \cdot \delta_{H}(p, q)$ for all points $p$ and $q$ in $S$. The smallest $t$ for which this property holds is called the stretch factor of $G$. Thus, a subgraph $G$ of $H$ with stretch factor $t$ approximates the $\left(\begin{array}{l}n \\ 2\end{array}\right)$ pairwise shortest-path lengths in $H$ within a factor of $t$. If $H$ is the complete geometric graph with vertex set $S$, then $G$ is also called a $t$-spanner of the point set $S$.

Most of the work on constructing spanners has been done for the case when $H$ is the complete graph. It is well known that for any set $S$ of $n$ points in $\mathbb{R}^{d}$ and for any real constant $\epsilon>0$, there exists a $(1+\epsilon)$-spanner of $S$ containing $O(n)$ edges. Moreover, such spanners can be computed in $O(n \log n)$ time; see Salowe [8] and Vaidya [9]. For a detailed overview of results on spanners for point sets, see the book by Narasimhan and Smid [6].

For spanners of arbitrary geometric graphs, much less is known. Althöfer et al. [1] have shown that for any $t>1$, every weighted graph $H$ with $n$ vertices contains a subgraph with $O\left(n^{1+2 /(t-1)}\right)$ edges, which is a $t$-spanner of $H$. Observe that this result holds for any weighted graph; in particular, it is valid for any geometric graph. For geometric graphs, a lower bound was given by Gudmundsson and Smid [5]: They proved that for every real number $t$ with $1<t<\frac{1}{4} \log n$, there exists a geometric graph $H$ with $n$ vertices, such that every $t$-spanner of $H$ contains $\Omega\left(n^{1+1 / t}\right)$ edges. Thus, if we are looking for spanners with $O(n)$ edges of arbitrary geometric graphs, then the best stretch factor we can obtain is $\Theta(\log n)$.

${ }^{*}$ School of Computer Science, Carleton University, Ottawa, Ontario, Canada K1S 5B6. Research partially supported by NSERC, MRI, CFI, and MITACS. 
In this paper, we consider the case when the input graph is a complete $k$-partite geometric graph. Let $S$ be a set of $n$ points in $\mathbb{R}^{d}$, and let $S$ be partitioned into subsets $C_{1}, C_{2}, \ldots, C_{k}$. Let $K_{C_{1} \ldots C_{k}}$ denote the complete $k$-partite graph on $S$. This graph has $S$ as its vertex set and two points $p$ and $q$ are connected by an edge (of length $|p q|$ ) if and only if $p$ and $q$ are in different subsets of the partition. The problem we address is formally defined as follows:

Problem 1.1 Let $k \geq 2$ be an integer, let $S$ be a set of $n$ points in $\mathbb{R}^{d}$, and let $S$ be partitioned into $k$ subsets $C_{1}, C_{2}, \ldots, C_{k}$. Compute a t-spanner of the complete $k$-partite graph $K_{C_{1} \ldots C_{k}}$ that has a "small" number of edges and whose stretch factor $t$ is "small".

The main contribution of this paper is to present an algorithm that computes such a $t$-spanner with $O(n)$ edges in $O(n \log n)$ time, where $t=5+\epsilon$ for any constant $\epsilon>0$. We also show that if one is willing to use $O(n \log n)$ edges, then our algorithm adapts easily to reach a stretch factor of $t=3+\epsilon$. Finally, we show that the latter result is optimal: For any $k$ with $2 \leq k \leq n-\Theta(\sqrt{n \log n})$, spanners with $O(n \log n)$ edges and stretch factor less than 3 do not exist for all complete $k$-partite geometric graphs.

We remark that in a recent paper, Bose et al. [2] considered the problem of constructing spanners of point sets that have $O(n)$ edges and whose chromatic number is a most $k$. This problem is different from ours: Bose et al. compute a spanner of the complete graph and their algorithm can choose a "good" $k$-partition of the vertices. In our problem, the $k$-partition is given and we want to compute a spanner of the complete $k$-partite graph.

Possible applications of our algorithm are in wireless networks having the property that communicating nodes are partitioned into sets such that two nodes can communicate if and only if they do not belong to the same set. This would be the case, for example, when Time Division Multiplexing (TDMA) is used. Since the wireless medium prohibits simultaneous transmission and reception at one node, two nodes communicating during the same time slots cannot communicate with each other; see Raman and Chebrolu [7].

The rest of this paper is organized as follows. In Section 2, we recall properties of the WellSeparated Pair Decomposition (WSPD) that we use in our algorithm. In Section 3, we provide an algorithm that solves the problem of constructing a spanner of the complete $k$-partite graph. In Section 4, we show that the spanner constructed by this algorithm has $O(n)$ edges and that its stretch factor is bounded from above by a constant that depends only on the dimension $d$. In Section 5, we show how a simple modification to our algorithm improves the stretch factor to $5+\epsilon$ while still having $O(n)$ edges. In Section 6, we show how to achieve a stretch factor of $3+\epsilon$ using $O(n \log n)$ edges. We also prove that the latter result is optimal. We conclude in Section 7 .

\section{The Well-Separated Pair Decomposition}

In this section, we recall crucial properties of the Well-Separated Pair Decomposition (WSPD) of Callahan and Kosaraju [4 that we use for our construction. The reader who is familiar with the WSPD may go directly to Section 3 . Our presentation follows the one in Narasimhan and Smid [6]. Intuitively, a WSPD is a partition of the edges of a complete geometric graph such that all edges that are grouped together are approximately equal. To give a formal definition of the WSPD, we first need to define what it means for two sets to be well-separated. 
Definition 2.1 Let $S$ be a set of points in $\mathbb{R}^{d}$. The bounding box $\beta(S)$ of $S$ is the smallest axes-parallel hyperrectangle that contains $S$.

Definition 2.2 Let $X$ and $Y$ be two sets of points in $\mathbb{R}^{d}$ and let $s>0$ be a real number. We say that $X$ and $Y$ are well-separated with respect to $s$ if there exists two balls $B_{1}$ and $B_{2}$ such that

1. $B_{1}$ and $B_{2}$ have the same radius, say $\rho$,

2. $B_{1}$ contains the bounding box of $X$,

3. $B_{2}$ contains the bounding box of $Y$, and

4. the distance $\min \left\{|x y|: x \in B_{1} \cap \mathbb{R}^{d}, y \in B_{2} \cap \mathbb{R}^{d}\right\}$ between $B_{1}$ and $B_{2}$ is at least s $\rho$.

Definition 2.3 Let $S$ be a set of points in $\mathbb{R}^{d}$ and let $s>0$ be a real number. A well-separated pair decomposition (WSPD) of $S$ with separation constant $s$ is a set of unordered pairs of subsets of $S$ that are well-separated with respect to $s$, such that for any two distinct points $p, q \in S$ there is a unique pair $\{X, Y\}$ in the WSPD such that $p \in X$ and $q \in Y$.

Lemma 2.4 (Lemma 9.1.2 in [6]) Let $s>0$ be a real number and let $X$ and $Y$ be two point sets that are well-separated with respect to $s$.

1. If $p, p^{\prime}, p^{\prime \prime} \in X$ and $q \in Y$, then $\left|p^{\prime} p^{\prime \prime}\right| \leq(2 / s)|p q|$.

2. If $p, p^{\prime} \in X$ and $q, q^{\prime} \in Y$, then $\left|p^{\prime} q^{\prime}\right| \leq(1+4 / s)|p q|$.

The first part of this lemma states that distances within one set are very small compared to distances between pairs of points having one endpoint in each set. The second part states that all pairs of points having one endpoint in each set have approximately the same distance.

Callahan and Kosaraju [3] have shown how to construct a $t$-spanner of $S$ from a WSPD: All one has to do is pick from each pair $\{X, Y\}$ an arbitrary edge $(p, q)$ with $p \in X$ and $q \in Y$. Using induction on the rank of the length of the edges in the complete graph $K_{S}$, it can be shown that, when $s>4$, this process leads to a $((s+4) /(s-4))$-spanner. Thus, by choosing $s$ to be a sufficiently large constant, the stretch factor can be made arbitrarily close to 1.

In order to compute a spanner of $S$ that has a linear number of edges, one needs a WSPD that has a linear number of pairs. Callahan and Kosaraju [4] showed that a WSPD with a linear number of pairs always exists and can be computed in time $O(n \log n)$. Their algorithm uses a split-tree.

Definition 2.5 Let $S$ be a non-empty set of points in $\mathbb{R}^{d}$. The split-tree of $S$ is defined as follows: if $S$ contains only one point, then the split-tree is a single node that stores that point. Otherwise, the split-tree has a root that stores the bounding box $\beta(S)$ of $S$, as well as an arbitrary point of $S$ called the representative of $S$ and denoted by $\operatorname{rep}(S)$. Split $\beta(S)$ into two hyperrectangles by cutting its longest interval into two equal parts, and let $S_{1}$ and $S_{2}$ be the subsets of $S$ contained in the two hyperrectangles. The root of the split-tree of $S$ has two sub-trees, which are recursively defined split-trees of $S_{1}$ and $S_{2}$.

The precise way Callahan and Kosaraju used the split-tree to compute a WSPD with a linear number of pairs is of no importance to us. The only important aspect we need to retain is that each pair is uniquely determined by a pair of nodes in the tree. More precisely, for each pair $\{X, Y\}$ 
in the WSPD that is output by their algorithm, there are unique internal nodes $u$ and $v$ in the split-tree such that the sets $S_{u}$ and $S_{v}$ of points stored at the leaves of the subtrees rooted at $u$ and $v$ are precisely $X$ and $Y$. Since there is such a unique correspondence, we will denote pairs in the WSPD by $\left\{S_{u}, S_{v}\right\}$, meaning that $u$ and $v$ are the nodes corresponding to the sets $X=S_{u}$ and $Y=S_{v}$. Also, although the WSPD of a point set is not unique, when we talk about the WSPD, we mean the WSPD that is computed by the algorithm of Callahan and Kosaraju.

Before we present our algorithm, we give the statement of the following lemmas that we use to analyze our algorithm in Section 4. If $R$ is an axes-parallel hyperrectangle in $\mathbb{R}^{d}$, then we use $L_{\max }(R)$ to denote the length of a longest side of $R$.

Lemma 2.6 (Lemma 9.5.3 in [6]) Let $u$ be a node in the split-tree and let $u^{\prime}$ be a node in the subtree of $u$ such that the path between them contains at least d edges. Then

$$
L_{\max }\left(\beta\left(S_{u^{\prime}}\right)\right) \leq \frac{1}{2} \cdot L_{\max }\left(\beta\left(S_{u}\right)\right) .
$$

Lemma 2.7 (Lemma 11.3.1 in [6]) Let $\left\{S_{u}, S_{v}\right\}$ be a pair in the WSPD, let $\ell$ be the distance between the centers of $\beta\left(S_{u}\right)$ and $\beta\left(S_{v}\right)$, and let $\pi(u)$ be the parent of $u$ in the split-tree. Then

$$
L_{\max }\left(\beta\left(S_{\pi(u)}\right)\right) \geq \frac{2 \ell}{\sqrt{d}(s+4)} .
$$

\section{A First Algorithm}

We now show how the WSPD can be used to address the problem of computing a spanner of a complete $k$-partite graph. In this section, we introduce an algorithm that outputs a graph with constant stretch factor and $O(n)$ edges. The analysis of this algorithm is presented in Section 4. In Section 5, we show how this algorithm can be improved to achieve a stretch factor of $5+\epsilon$.

The input set $S \subseteq \mathbb{R}^{d}$ is the disjoint union of $k$ sets $C_{1}, C_{2}, \ldots, C_{k}$. We say that the elements of $C_{c}$ have "color" $c$. The graph $K=K_{C_{1} \ldots C_{k}}$ is the complete $k$-partite geometric graph.

Definition 3.1 Let $T$ be the split-tree of $S$ that is used to compute the WSPD of $S$.

1. For any node $u$ in $T$, we denote by $S_{u}$ the set of all points in the subtree rooted at $u$.

2. We define MWSPD to be the subset of the WSPD obtained by removing all pairs $\left\{S_{u}, S_{v}\right\}$ for which all points of $S_{u} \cup S_{v}$ have the same color.

3. A node $u$ in $T$ is called multichromatic if there exist points $p$ and $q$ in $S_{u}$ and a node $v$ in $T$, such that $p$ and $q$ have different colors and $\left\{S_{u}, S_{v}\right\}$ is in the MWSPD.

4. A node $u$ in $T$ is called a $c$-node if all points of $S_{u}$ have color $c$ and there exists a node $v$ in $T$ such that $\left\{S_{u}, S_{v}\right\}$ is in the MWSPD.

5. A c-node $u$ in $T$ is called a c-root if it does not have a proper ancestor that is a c-node in $T$.

6. A c-node $u$ in $T$ is called a c-leaf if it does not have another c-node in its subtree.

7. A c-node $u^{\prime}$ in $T$ is called a $c$-child of a c-node $u$ in $T$ if $u^{\prime}$ is in the subtree rooted at $u$ and there is no c-node on the path strictly between $u$ and $u^{\prime}$. 


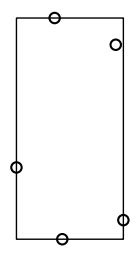

All points are of the same color.

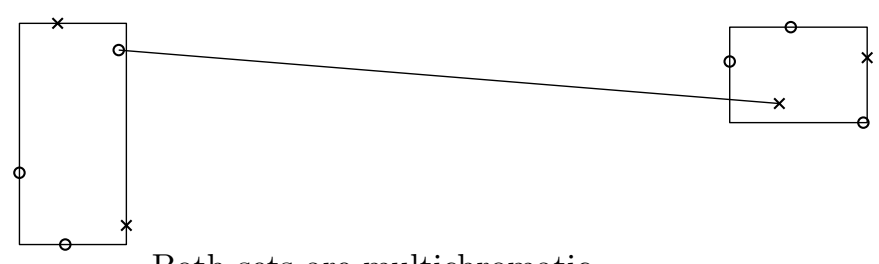

Both sets are multichromatic.

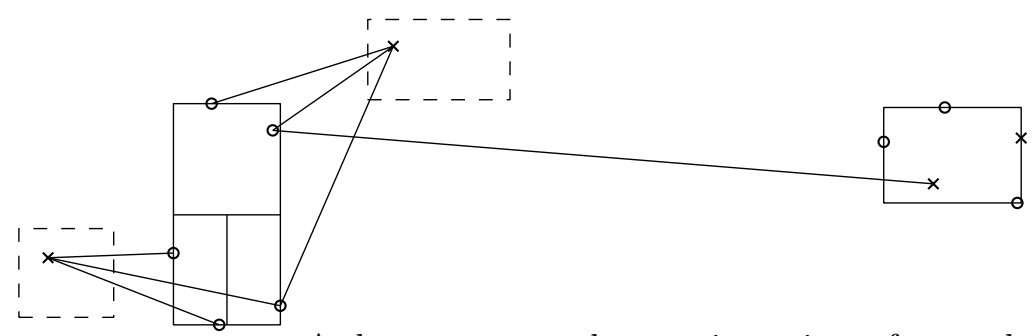

At least one set only contains points of one color but not all points are of the same color.

Figure 1: The three cases of Algorithm 1.

8. For each color $c$ and for each c-node $u$ in $T, \operatorname{rep}\left(S_{u}\right)$ denotes a fixed arbitrary point in $S_{u}$.

9. For each multichromatic node $u$ in $T, \operatorname{rep}\left(S_{u}\right)$ and $r e p^{\prime}\left(S_{u}\right)$ denote two fixed arbitrary points in $S_{u}$ that have different colors.

10. The distance between two sets $S_{v}$ and $S_{w}$, denoted by $\operatorname{dist}\left(S_{v}, S_{w}\right)$, is defined to be the distance between the centers of their bounding boxes.

11. Let $u$ be a c-node in $T$. Consider all pairs $\left\{S_{v}, S_{w}\right\}$ in the $M W S P D$, where $v$ is a c-node on the path in $T$ from $u$ to the root (this path includes $u$ ). Let $\left\{S_{v}, S_{w}\right\}$ be such a pair for which $\operatorname{dist}\left(S_{v}, S_{w}\right)$ is minimum. We define $\operatorname{cl}\left(S_{u}\right)$ to be the set $S_{w}$.

Algorithm 1 computes a spanner of a complete $k$-partite geometric graph $K=K_{C_{1} \ldots C_{k}}$. The intuition behind this algorithm is the following. First, the algorithm computes the WSPD. Then, it considers each pair $\left\{S_{u}, S_{v}\right\}$ of the WSPD, and decides whether or not to add an edge between $S_{u}$ and $S_{v}$. The outcome of this decision is based on the following three cases, which are illustrated in Figures 1 and 2 .

Case 1: All points of $S_{u} \cup S_{v}$ are of the same color. In this case, there is no edge of $K$ to approximate, so the algorithm ignores this pair.

Case 2: Both $S_{u}$ and $S_{v}$ are multichromatic. In this case, the algorithm adds one edge between 


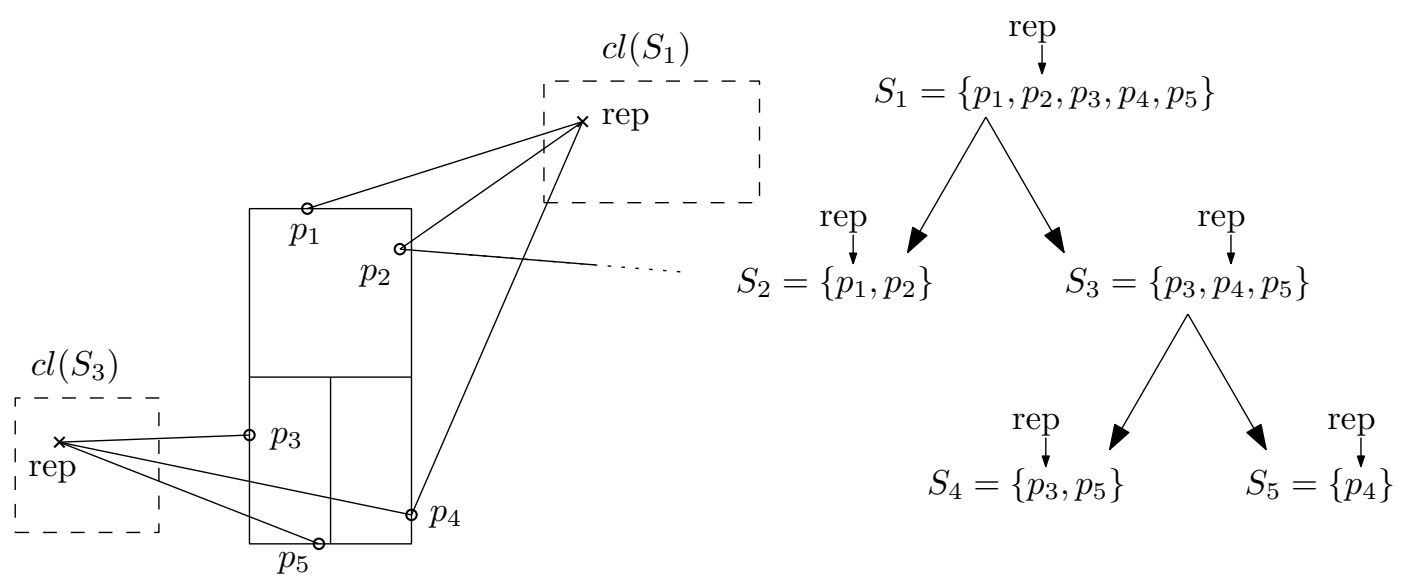

Figure 2: Handling a $c$-node.

$S_{u}$ and $S_{v}$ to the spanner; see lines 28,29 . Observe that the two vertices of this edge do not have the same color. This edge will allow us to approximate each edge $(p, q)$ of $K$, where $p \in S_{u}, q \in S_{v}$, and $p$ and $q$ have different colors.

Case 3: All points in $S_{u}$ are of the same color $c$. In this case, an edge is added between $\operatorname{rep}\left(S_{u}\right)$ and one of the two representatives of $S_{v}$ whose color is not $c$; see lines 17,18 , In order to approximate each edge of $K$ having one vertex (of color $c$ ) in $S_{u}$ and the other vertex (of a different color) in $S_{v}$, more edges have to be added. This is done in such a way that our final graph contains a "short" path between every point $p$ of $S_{u}$ and the representative $\operatorname{rep}\left(S_{u}\right)$ of $S_{u}$. Observe that this path must contain points whose color is not equal to $c$; thus, these points are not in $S_{u}$. One way to achieve this is to add an edge between each point of $S_{u}$ and one of the two representatives of $\operatorname{cl}\left(S_{u}\right)$ whose color is not $c$; we call this construction a star. However, since the subtree rooted at $u$ may contain other $c$-nodes, many edges may be added for each point in $S_{u}$, which could possibly lead to a quadratic number of edges in the final graph. To guarantee that the algorithm does not add too many edges, it introduces a star only if $u$ is a $c$-leaf; see lines 811. If $u$ is a $c$-node, the algorithm only adds one edge between $\operatorname{rep}\left(S_{u}\right)$ and a representatives of $\operatorname{cl}\left(S_{u}\right)$ whose color is not $c$; see lines 14 15. Then, the algorithm links each $c$-node $u^{\prime \prime}$ that is not a $c$-root to its $c$-parent $u^{\prime}$. This is done through an edge between $\operatorname{rep}\left(S_{u^{\prime \prime}}\right)$ and a representative of $\operatorname{cl}\left(S_{u^{\prime}}\right)$ whose color is not $c$; see lines 2122 . This third case is illustrated in Figure 2.

\section{Analysis of Algorithm 1}

Lemma 4.1 The graph $G$ computed by Algorithm 1 has $O(|S|)$ edges.

Proof: For each color $c$ and for each $c$-leaf $u^{\prime}$, the algorithm adds $\left|S_{u^{\prime}}\right|$ edges to $G$ in lines 9410 . Since the sets $S_{u^{\prime}}$, where $u^{\prime}$ ranges over all $c$-leaves and $c$ ranges over all colors, are pairwise disjoint, the total number of edges that are added in lines $9 \sqrt{10}$ is $O(|S|)$.

The total number of edges that are added in lines 17,18 and 2829 is at most the number of pairs in the MWSPD. Since the WSPD contains $O(|S|)$ pairs (see [4]), the same upper bound holds for the number of edges added in lines 17,18 and $28,29$.

The total number of edges that are added in lines 14,15 and 21,22 is at most twice the number 


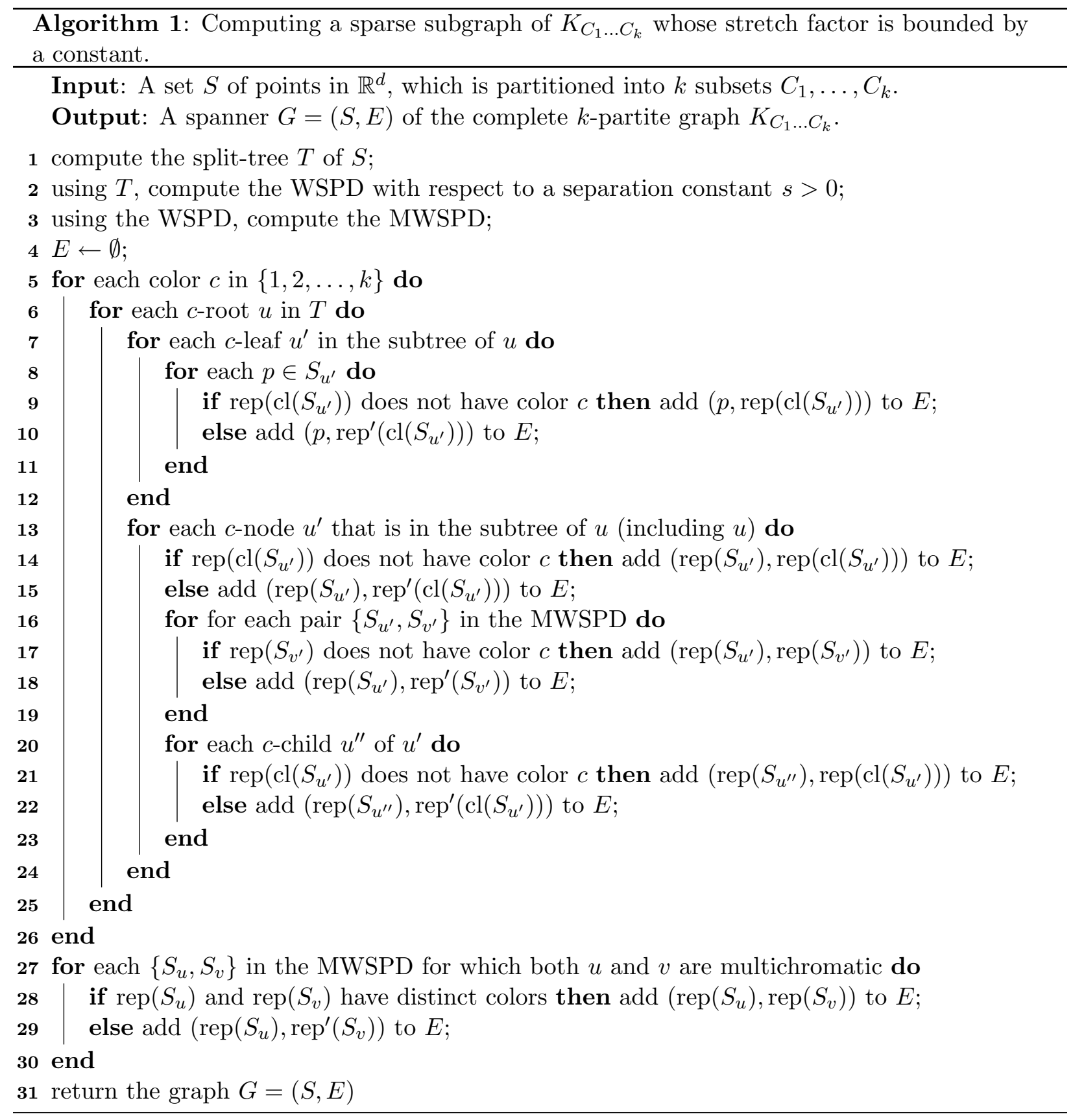


of nodes in the split-tree, which is $O(|S|)$.

Lemma 4.2 Let $G$ be the graph computed by Algorithm 1. Let $p$ and $q$ be two points of $S$ with different colors, and let $\left\{S_{u}, S_{v}\right\}$ be the pair in the MWSPD for which $p \in S_{u}$ and $q \in S_{v}$. Assume that $u$ is a c-node for some color $c$. Then there is a path in $G$ between $p$ and $\operatorname{rep}\left(S_{u}\right)$ whose length is at most $t^{\prime}|p q|$, where

$$
\begin{aligned}
& t^{\prime}=4 \sqrt{d}(\mu d+1)(1+4 / s)^{3}, \\
& \mu=\lceil\log (\sqrt{d}(1+4 / s))]+1,
\end{aligned}
$$

and $s$ is the separation constant of the WSPD.

Proof: Let $w$ be the $c$-leaf such that $p \in S_{w}$, and let $w=w_{0}, \ldots, w_{k}=u$ be the sequence of $c$-nodes that are on the path in $T$ from $w$ to $u$.

Recall from Definition 3.1 that each set $S_{w_{i}}, 0 \leq i \leq k$, has a representative $\operatorname{rep}\left(S_{w_{i}}\right)$ (of color c) associated with it. Also, recall the definition of the sets $\operatorname{cl}\left(S_{w_{i}}\right), 0 \leq i \leq k$; see Definition 3.1 If $\operatorname{cl}\left(S_{w_{i}}\right)$ is a $c^{\prime}$-node for some color $c^{\prime}$, then this set has one representative $\operatorname{rep}\left(\operatorname{cl}\left(S_{w_{i}}\right)\right)$ associated with it. Otherwise, $\operatorname{cl}\left(S_{w_{i}}\right)$ is multichromatic and this set has two representatives $\operatorname{rep}\left(\operatorname{cl}\left(S_{w_{i}}\right)\right)$ and $\operatorname{rep}^{\prime}\left(\operatorname{cl}\left(S_{w_{i}}\right)\right)$ of different colors associated with it. We may assume without loss of generality that, for all $0 \leq i \leq k$, the color of $\operatorname{rep}\left(\operatorname{cl}\left(S_{w_{i}}\right)\right)$ is not equal to $c$.

Let $\Pi$ be the path

$$
\begin{aligned}
& p \rightarrow \operatorname{rep}\left(\operatorname{cl}\left(S_{w_{0}}\right)\right) \rightarrow \operatorname{rep}\left(S_{w_{0}}\right) \\
& \rightarrow \operatorname{rep}\left(\operatorname{cl}\left(S_{w_{1}}\right)\right) \rightarrow \operatorname{rep}\left(S_{w_{1}}\right) \\
& \vdots \quad \vdots \\
& \rightarrow \operatorname{rep}\left(\operatorname{cl}\left(S_{w_{k}}\right)\right) \rightarrow \operatorname{rep}\left(S_{w_{k}}\right)=\operatorname{rep}\left(S_{u}\right) .
\end{aligned}
$$

The first edge on this path, i.e., $\left(p, \operatorname{rep}\left(\operatorname{cl}\left(S_{w_{0}}\right)\right)\right)$, is added to the graph $G$ in lines $9 \sqrt{10}$ of the algorithm. The edges $\left(\operatorname{rep}\left(\operatorname{cl}\left(S_{w_{i}}\right)\right), \operatorname{rep}\left(S_{w_{i}}\right)\right), 0 \leq i \leq k$, are added to $G$ in lines 14 15. Finally, the edges $\left(\operatorname{rep}\left(S_{w_{i-1}}\right), \operatorname{rep}\left(\operatorname{cl}\left(S_{w_{i}}\right)\right)\right), 1 \leq i \leq k$, are added to $G$ in lines $21 \sqrt{22}$. It follows that $\Pi$ is a path in $G$ between $p$ and $\operatorname{rep}\left(S_{u}\right)$. We will show that the length of $\Pi$ is at most $t^{\prime}|p q|$.

Let $i$ be an integer with $0 \leq i \leq k$. Recall the definition of $\operatorname{cl}\left(S_{w_{i}}\right)$; see Definition 3.1. We consider all pairs $\left\{S_{x}, S_{y}\right\}$ in the MWSPD, where $x$ is a $c$-node on the path in $T$ from $w_{i}$ to the root, and pick the pair for which $\operatorname{dist}\left(S_{x}, S_{y}\right)$ is minimum. We denote the pair picked by $\left(S_{x_{i}}, S_{y_{i}}\right)$. Thus, $x_{i}$ is a $c$-node on the path in $T$ from $w_{i}$ to the root, $\left\{S_{x_{i}}, S_{y_{i}}\right\}$ is a pair in the MWSPD, and $\operatorname{cl}\left(S_{w_{i}}\right)=S_{y_{i}}$. We define

$$
\ell_{i}=\operatorname{dist}\left(S_{x_{i}}, S_{y_{i}}\right) \text {. }
$$

Consider the first edge $\left(p, \operatorname{rep}\left(\operatorname{cl}\left(S_{w_{0}}\right)\right)\right)$ on the path $\Pi$. Since $p \in S_{w_{0}} \subseteq S_{x_{0}}$ and $\operatorname{rep}\left(\operatorname{cl}\left(S_{w_{0}}\right)\right) \in$ $S_{y_{0}}$, it follows from Lemma 2.4 that

$$
\left|p, \operatorname{rep}\left(\operatorname{cl}\left(S_{w_{0}}\right)\right)\right| \leq(1+4 / s) \cdot \operatorname{dist}\left(S_{x_{0}}, S_{y_{0}}\right)=(1+4 / s) \ell_{0} .
$$

Let $0 \leq i \leq k$ and consider the edge $\left(\operatorname{rep}\left(\operatorname{cl}\left(S_{w_{i}}\right)\right), \operatorname{rep}\left(S_{w_{i}}\right)\right)$ on $\Pi$. Since $\operatorname{rep}\left(S_{w_{i}}\right) \in S_{w_{i}} \subseteq S_{x_{i}}$ and $\operatorname{rep}\left(\operatorname{cl}\left(S_{w_{i}}\right)\right) \in S_{y_{i}}$, it follows from Lemma 2.4 that

$$
\left|\operatorname{rep}\left(\operatorname{cl}\left(S_{w_{i}}\right)\right), \operatorname{rep}\left(S_{w_{i}}\right)\right| \leq(1+4 / s) \cdot \operatorname{dist}\left(S_{x_{i}}, S_{y_{i}}\right)=(1+4 / s) \ell_{i} .
$$


Let $1 \leq i \leq k$ and consider the edge $\left(\operatorname{rep}\left(S_{w_{i-1}}\right), \operatorname{rep}\left(\operatorname{cl}\left(S_{w_{i}}\right)\right)\right)$ on $\Pi$. Since $\operatorname{rep}\left(S_{w_{i-1}}\right) \in S_{w_{i-1}} \subseteq S_{x_{i}}$ and $\operatorname{rep}\left(\operatorname{cl}\left(S_{w_{i}}\right)\right) \in S_{y_{i}}$, it follows from Lemma 2.4 that

$$
\left|\operatorname{rep}\left(S_{w_{i-1}}\right), \operatorname{rep}\left(\operatorname{cl}\left(S_{w_{i}}\right)\right)\right| \leq(1+4 / s) \cdot \operatorname{dist}\left(S_{x_{i}}, S_{y_{i}}\right)=(1+4 / s) \ell_{i} .
$$

Thus, the length of the path $\Pi$ is at most

$$
\sum_{i=0}^{k} 2(1+4 / s) \ell_{i}
$$

Therefore, it is sufficient to prove that

$$
\sum_{i=0}^{k} \ell_{i} \leq 2 \sqrt{d}(\mu d+1)(1+4 / s)^{2}|p q| .
$$

It follows from the definition of $\operatorname{cl}\left(S_{u}\right)=\operatorname{cl}\left(S_{w_{k}}\right)$ that

$$
\ell_{k}=\operatorname{dist}\left(S_{x_{k}}, S_{y_{k}}\right) \leq \operatorname{dist}\left(S_{u}, S_{v}\right)
$$

Since, by Lemma 2.4 $\operatorname{dist}\left(S_{u}, S_{v}\right) \leq(1+4 / s)|p q|$, it follows that

(2) $\quad \ell_{k} \leq(1+4 / s)|p q|$.

Thus, it is sufficient to prove that

$$
\sum_{i=0}^{k} \ell_{i} \leq 2 \sqrt{d}(\mu d+1)(1+4 / s) \ell_{k}
$$

If $k=0$, then (3) obviously holds. Assume from now on that $k \geq 1$. For each $i$ with $0 \leq i \leq k$, we define

$$
a_{i}=L_{\max }\left(\beta\left(S_{w_{i}}\right)\right)
$$

i.e., $a_{i}$ is the length of a longest side of the bounding box of $S_{w_{i}}$.

Let $0 \leq i \leq k$. It follows from Lemma 2.4 that

$$
L_{\max }\left(\beta\left(S_{x_{i}}\right)\right) \leq \frac{2}{s} \ell_{i} .
$$

Since $w_{i}$ is in the subtree of $x_{i}$, we have $L_{\max }\left(\beta\left(S_{w_{i}}\right)\right) \leq L_{\max }\left(\beta\left(S_{x_{i}}\right)\right)$. Thus, we have

$$
a_{i} \leq \frac{2}{s} \ell_{i} \text { for } 0 \leq i \leq k .
$$

Lemma 2.6 states that

$$
a_{i} \leq \frac{1}{2} a_{i+d} \text { for } 0 \leq i \leq k-d .
$$

Let $0 \leq i \leq k-1$. Since $w_{i}$ is a $c$-node, there is a node $w_{i}^{\prime}$ such that $\left\{S_{w_{i}}, S_{w_{i}^{\prime}}\right\}$ is a pair in the MWSPD. Then it follows from the definition of $\operatorname{cl}\left(S_{w_{i}}\right)$ that

$$
\ell_{i}=\operatorname{dist}\left(S_{x_{i}}, S_{y_{i}}\right) \leq \operatorname{dist}\left(S_{w_{i}}, S_{w_{i}^{\prime}}\right) .
$$


By applying Lemma 2.7, we obtain

$$
\begin{aligned}
\operatorname{dist}\left(S_{w_{i}}, S_{w_{i}^{\prime}}\right) & \leq \frac{\sqrt{d}(s+4)}{2} L_{\max }\left(\beta\left(S_{\pi\left(w_{i}\right)}\right)\right) \\
& \leq \frac{\sqrt{d}(s+4)}{2} L_{\max }\left(\beta\left(S_{w_{i+1}}\right)\right) \\
& =\frac{\sqrt{d}(s+4)}{2} a_{i+1} .
\end{aligned}
$$

Thus, we have

(6) $\quad \ell_{i} \leq \frac{\sqrt{d}(s+4)}{2} a_{i+1}$ for $0 \leq i \leq k-1$.

First assume that $1 \leq k \leq \mu d$. Let $0 \leq i \leq k-1$. By using (6), the fact that the sequence $a_{0}, a_{1}, \ldots, a_{k}$ is non-decreasing, and (4), we obtain

$$
\ell_{i} \leq \frac{\sqrt{d}(s+4)}{2} a_{i+1} \leq \frac{\sqrt{d}(s+4)}{2} a_{k} \leq \sqrt{d}(1+4 / s) \ell_{k} .
$$

Therefore,

$$
\sum_{i=0}^{k} \ell_{i} \leq k \sqrt{d}(1+4 / s) \ell_{k}+\ell_{k} \leq(k+1) \sqrt{d}(1+4 / s) \ell_{k} \leq(\mu d+1) \sqrt{d}(1+4 / s) \ell_{k},
$$

which is less than the right-hand side in (3).

It remains to consider the case when $k>\mu d$. Let $i \geq 0$ and $j \geq 0$ be integers such that $i+1+j d \leq k$. By applying (6) once, (5) $j$ times, and (4) once, we obtain

$$
\ell_{i} \leq \frac{\sqrt{d}(s+4)}{2} a_{i+1} \leq \frac{\sqrt{d}(s+4)}{2}\left(\frac{1}{2}\right)^{j} a_{i+1+j d} \leq \sqrt{d}(1+4 / s)\left(\frac{1}{2}\right)^{j} \ell_{i+1+j d}
$$

For $j=\mu=\lceil\log (\sqrt{d}(1+4 / s))\rceil+1$, this implies that, for $0 \leq i \leq k-1-\mu d$,

$$
\ell_{i} \leq \frac{1}{2} \ell_{i+1+\mu d}
$$

By re-arranging the terms in the summation in (3), we obtain

$$
\sum_{i=0}^{k} \ell_{i}=\sum_{h=0}^{\mu d} \sum_{j=0}^{\lfloor(k-h) /(\mu d+1)\rfloor} \ell_{k-h-j(\mu d+1)}
$$

Let $j$ be such that $0 \leq j \leq\lfloor(k-h) /(\mu d+1)\rfloor$. By applying (7) $j$ times, we obtain

$$
\ell_{k-h-j(\mu d+1)} \leq\left(\frac{1}{2}\right)^{j} \ell_{k-h}
$$

It follows that

$$
\sum_{j=0}^{\lfloor(k-h) /(\mu d+1)\rfloor} \ell_{k-h-j(\mu d+1)} \leq \sum_{j=0}^{\infty}\left(\frac{1}{2}\right)^{j} \ell_{k-h}=2 \ell_{k-h} .
$$


Thus, we have

$$
\sum_{i=0}^{k} \ell_{i} \leq 2 \sum_{h=0}^{\mu d} \ell_{k-h}
$$

By applying (6), the fact that the sequence $a_{0}, a_{1}, \ldots, a_{k}$ is non-decreasing, followed by (4), we obtain, for $0 \leq i \leq k-1$ and $1 \leq j \leq k-i$,

$$
\ell_{i} \leq \frac{\sqrt{d}(s+4)}{2} a_{i+1} \leq \frac{\sqrt{d}(s+4)}{2} a_{i+j} \leq \sqrt{d}(1+4 / s) \ell_{i+j}
$$

Obviously, the inequality $\ell_{i} \leq \sqrt{d}(1+4 / s) \ell_{i+j}$ also holds for $j=0$. Thus, for $i=k-h$ and $j=h$, we get

$$
\ell_{k-h} \leq \sqrt{d}(1+4 / s) \ell_{k} \text { for } 0 \leq h \leq \mu d .
$$

It follows that

$$
\sum_{i=0}^{k} \ell_{i} \leq 2 \sum_{h=0}^{\mu d} \sqrt{d}(1+4 / s) \ell_{k}=2 \sqrt{d}(\mu d+1)(1+4 / s) \ell_{k},
$$

completing the proof that (3) holds.

Lemma 4.3 Assuming that the separation constant s of the WSPD is chosen sufficiently large, the graph $G$ computed by Algorithm 1 is a t-spanner of the complete $k$-partite graph $K_{C_{1} \ldots C_{k}}$, where $t=2 t^{\prime}+1+4 / s$ and $t^{\prime}$ is as in Lemma 4.2.

Proof: We denote the graph $K_{C_{1} \ldots C_{k}}$ by $K$. It suffices to show that for each edge $(p, q)$ of $K$, the graph $G$ contains a path between $p$ and $q$ of length at most $t|p q|$. We will prove this by induction on the lengths of the edges in $K$.

Let $p$ and $q$ be two points of $S$ with different colors, and let $\left\{S_{u}, S_{v}\right\}$ be the pair in the MWSPD for which $p \in S_{u}$ and $q \in S_{v}$.

The base case is when $(p, q)$ is a shortest edge in $K$. Since $s>2$, it follows from Lemma 2.4 that $u$ is a $c$-node and $v$ is a $c^{\prime}$-node, for some colors $c$ and $c^{\prime}$ with $c \neq c^{\prime}$. In line 17 of Algorithm 1 , the edge $\left(\operatorname{rep}\left(S_{u}\right), \operatorname{rep}\left(S_{v}\right)\right)$ is added to $G$. By Lemma 2.4 , the length of this edge is at most $(1+4 / s)|p q|$. The claim follows from two applications of Lemma 4.2 to get from $p$ to $\operatorname{rep}\left(S_{u}\right)$ and from $\operatorname{rep}\left(S_{v}\right)$ to $q$.

In the induction step, we distinguish four cases.

Case 1: $u$ is a $c$-node and $v$ is a $c^{\prime}$-node, for some colors $c$ and $c^{\prime}$ with $c \neq c^{\prime}$.

This case is identical to the base case.

Case 2: $u$ is a $c$-node for some color $c$ and $v$ is a multichromatic node.

In lines 17,18 , the edge $\left(\operatorname{rep}\left(S_{u}\right), \operatorname{rep}\left(S_{v}\right)\right)$ or $\left(\operatorname{rep}\left(S_{u}\right), \operatorname{rep}\left(S_{v}^{\prime}\right)\right)$ is added to $G$. We may assume without loss of generality that $\left(\operatorname{rep}\left(S_{u}\right), \operatorname{rep}\left(S_{v}\right)\right)$ is added. By Lemma 2.4, the length of this edge is at most $(1+4 / s)|p q|$.

By Lemma 4.2, there is a path in $G$ between $p$ and $\operatorname{rep}\left(S_{u}\right)$ whose length is at most $t^{\prime}|p q|$. 
First assume that $q$ and $\operatorname{rep}\left(S_{v}\right)$ have the same color. Let $r$ be a point in $S_{v}$ that has a color different from $q$ 's color. Since $s>2$, it follows from Lemma 2.4 that $|q r|<|p q|$. Thus, by induction, there is a path in $G$ between $q$ and $r$ whose length is at most $t|q r|$, which, by Lemma 2.4 , is at most $(2 t / s)|p q|$. By a similar argument, since $\left|r, \operatorname{rep}\left(S_{v}\right)\right|<|p q|$, there is a path in $G$ between $r$ and $\operatorname{rep}\left(S_{v}\right)$ whose length is at most $(2 t / s)|p q|$. Thus, $G$ contains a path between $q$ and $\operatorname{rep}\left(S_{v}\right)$ of length at most $(4 t / s)|p q|$. If $q$ and $\operatorname{rep}\left(S_{v}\right)$ have different colors, then, by induction, there is a path in $G$ between $q$ and $\operatorname{rep}\left(S_{v}\right)$ whose length is at most $(2 t / s)|p q|<(4 t / s)|p q|$.

Thus, the graph $G$ contains a path between $q$ and $\operatorname{rep}\left(S_{v}\right)$ of length at most $(4 t / s)|p q|$.

We have shown that there is a path in $G$ between $p$ and $q$ whose length is at most

$$
\left(t^{\prime}+(1+4 / s)+4 t / s\right)|p q| \text {. }
$$

By choosing sufficiently large, this quantity is at most $t|p q|$.

Case 3: $u$ is a multichromatic node and $v$ is a $c$-node for some color $c$.

This case is symmetric to Case 2 .

Case 4: Both $u$ and $v$ are multichromatic nodes.

In lines 2829 , the edge $\left(\operatorname{rep}\left(S_{u}\right), \operatorname{rep}\left(S_{v}\right)\right)$ or $\left(\operatorname{rep}\left(S_{u}\right), \operatorname{rep}\left(S_{v}^{\prime}\right)\right)$ is added to $G$. We may assume without loss of generality that $\left(\operatorname{rep}\left(S_{u}\right), \operatorname{rep}\left(S_{v}\right)\right)$ is added. By Lemma 2.4, the length of this edge is at most $(1+4 / s)|p q|$.

As in Case 2, the graph $G$ contains a path between $p$ and $\operatorname{rep}\left(S_{u}\right)$ of length at most $(4 t / s)|p q|$, and a path between $q$ and $\operatorname{rep}\left(S_{v}\right)$ of length at most $(4 t / s)|p q|$.

It follows that there is a path in $G$ between $p$ and $q$ whose length is at most

$$
((1+4 / s)+8 t / s)|p q| \text {. }
$$

By choosing $s$ sufficiently large, this quantity is at most $t|p q|$.

Lemma 4.4 The running time of Algorithm 1 is $O(n \log n)$, where $n=|S|$.

Proof: Using the results of Callahan and Kosaraju [4], the split-tree $T$ and the WSPD can be computed in $O(n \log n)$ time. The representatives of all sets $S_{u}$ and all sets $\operatorname{cl}\left(S_{u}\right)$ can be computed in $O(n)$ time by traversing the split-tree in post-order and pre-order, respectively. The time for the rest of the algorithm, i.e., lines 3 31, is proportional to the sum of the size of $T$, the number of pairs in the WSPD and the number of edges in the graph $G$. Thus, the rest of the algorithm takes $O(n)$ time.

To summarize, we have shown the following: For any complete $k$-partite geometric graph $K$ whose vertex set has size $n$, Algorithm 1 computes a $t$-spanner of $K$ having $O(n)$ edges, where $t$ is given in Lemma 4.3. The running time of this algorithm is $O(n \log n)$. By choosing the separation constant $s$ sufficiently large, the stretch factor $t$ converges to

$$
8 \sqrt{d}\left(d\left\lceil\frac{1}{2} \log d\right\rceil+d+1\right)+1
$$

In the next section, we show how to modify the algorithm so that the bound in Lemma 4.2 is reduced, thus improving the stretch factor. The price to pay is in the number of edges in $G$, however, it is still $O(n)$. 


\section{An Improved Algorithm}

As before, we are given a set $S$ of $n$ points in $\mathbb{R}^{d}$ which is partitioned into $k$ subsets $C_{1}, C_{2}, \ldots, C_{k}$. Intuitively, the way to improve the bound of Lemma 4.2 is by adding shortcuts along the path from each $c$-leaf to the $c$-root above it. More precisely, from (7) in the proof of Lemma 4.2, we know that if we go $1+\mu d$ levels up in the split-tree $T$, then the length of the edge along the path doubles. Thus, for each $c$-node in $T$, we will add edges to all $2 \delta(1+\mu d) c$-nodes above it in $T$. Here, $\delta$ is an integer constant that is chosen such that the best result is obtained in the improved bound.

Definition 5.1 Let $c \in\{1,2, \ldots, k\}$, and let $u$ and $u^{\prime}$ be c-nodes in the split-tree $T$ such that $u^{\prime}$ is in the subtree rooted at $u$. For any integer $\zeta \geq 1$, we say that $u$ is $\zeta$ levels above $u^{\prime}$, if there are exactly $\zeta-1$ c-nodes on the path strictly between $u$ and $u^{\prime}$. We say that $u^{\prime}$ is a $\zeta$-level $c$-child of $u$ if $u$ is at most $\zeta$ levels above $u^{\prime}$.

The improved algorithm is given as Algorithm 2. The following lemma generalizes Lemma 4.2 .

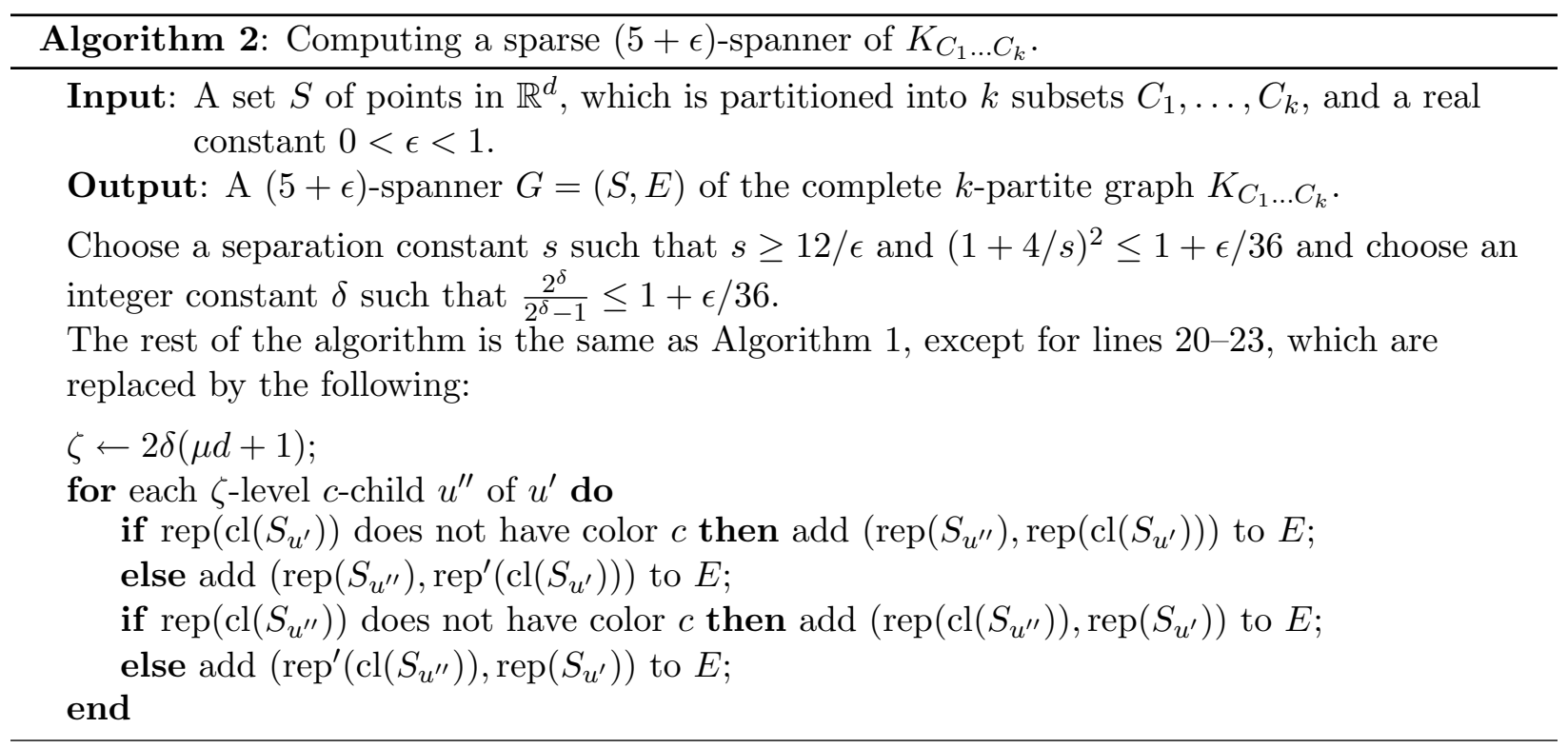

Lemma 5.2 Let $G$ be the graph computed by Algorithm 2. Let $p$ and $q$ be two points of $S$ with different colors, and let $\left\{S_{u}, S_{v}\right\}$ be the pair in the MWSPD for which $p \in S_{u}$ and $q \in S_{v}$. Assume that $u$ is a c-node for some color $c$. Then there is a path in $G$ between $p$ and $\operatorname{rep}\left(S_{u}\right)$ whose length is at most $(2+\epsilon / 3)|p q|$.

Proof: Let $w$ be the $c$-leaf such that $r \in S_{w}$, and let $w=w_{0}, w_{1}, \ldots, w_{k}=u$ be the sequence of $c$-nodes that are on the path in $T$ from $w$ to $u$. As in the proof of Lemma 4.2 , we assume without loss of generality that, for all $0 \leq i \leq k$, the color of $\operatorname{rep}\left(\operatorname{cl}\left(S_{w_{i}}\right)\right)$ is not equal to $c$.

Throughout the proof, we will use the variables $x_{i}, y_{i}, \ell_{i}$, and $a_{i}$, for $0 \leq i \leq k$, that were introduced in the proof of Lemma 4.2 .

We first assume that $0 \leq k \leq 2 \delta(\mu d+1)$. Let $\Pi$ be the path

$$
p \rightarrow \operatorname{rep}\left(\operatorname{cl}\left(S_{w}\right)\right) \rightarrow \operatorname{rep}\left(S_{u}\right)
$$


It follows from Algorithm 2 that $\Pi$ is a path in $G$. Since $p \in S_{w}=S_{w_{0}} \subseteq S_{x_{0}}$ and $\operatorname{rep}\left(\operatorname{cl}\left(S_{w}\right)\right)=$ $\operatorname{rep}\left(\operatorname{cl}\left(S_{w_{0}}\right)\right) \in S_{y_{0}}$, it follows from Lemma 2.4 that

$$
\left|p, \operatorname{rep}\left(\operatorname{cl}\left(S_{w}\right)\right)\right| \leq(1+4 / s) \cdot \operatorname{dist}\left(S_{x_{0}}, S_{y_{0}}\right)=(1+4 / s) \ell_{0} .
$$

Since $\left\{S_{u}, S_{v}\right\}$ is one of the pairs that is considered in the definition of $\operatorname{cl}\left(S_{w_{0}}\right)$, we have $\operatorname{dist}\left(S_{x_{0}}, S_{y_{0}}\right) \leq$ $\operatorname{dist}\left(S_{u}, S_{v}\right)$. Again by Lemma 2.4, we have $\operatorname{dist}\left(S_{u}, S_{v}\right) \leq(1+4 / s)|p q|$. Thus, we have shown that

$$
\left|p, \operatorname{rep}\left(\operatorname{cl}\left(S_{w}\right)\right)\right| \leq(1+4 / s)^{2}|p q| .
$$

By the triangle inequality, we have

$$
\left|\operatorname{rep}\left(\operatorname{cl}\left(S_{w}\right)\right), \operatorname{rep}\left(S_{u}\right)\right| \leq\left|\operatorname{rep}\left(\operatorname{cl}\left(S_{w}\right)\right), p\right|+\left|p, \operatorname{rep}\left(S_{u}\right)\right|
$$

Since $p$ and $\operatorname{rep}\left(S_{u}\right)$ are both contained in $S_{u}$, it follows from Lemma 2.4 that $\left|p, \operatorname{rep}\left(S_{u}\right)\right| \leq$ $(2 / s)|p q|$. Thus, we have

$$
\left|\operatorname{rep}\left(\operatorname{cl}\left(S_{w}\right)\right), \operatorname{rep}\left(S_{u}\right)\right| \leq(1+4 / s)^{2}|p q|+(2 / s)|p q| .
$$

We have shown that the length of the path $\Pi$ is at most

$$
\left(2(1+4 / s)^{2}+2 / s\right)|p q|,
$$

which is at most $(2+\epsilon / 3)|p q|$ by our choice of $s$ in Algorithm 2 .

In the rest of the proof, we assume that $k>2 \delta(\mu d+1)$. We define

$$
m=k \bmod (\delta(\mu d+1))
$$

and

$$
m^{\prime}=\frac{k-m}{\delta(\mu d+1)} .
$$

We consider the sequence of $c$-nodes

$$
w=w_{0}, w_{\delta(\mu d+1)+m}, w_{2 \delta(\mu d+1)+m}, w_{3 \delta(\mu d+1)+m}, \ldots, w_{k}=u,
$$

and define $\Pi$ to be the path

$$
\begin{aligned}
& p \rightarrow \operatorname{rep}\left(\operatorname{cl}\left(S_{w_{0}}\right)\right) \quad \rightarrow \quad \operatorname{rep}\left(S_{w_{\delta(\mu d+1)+m}}\right) \\
& \rightarrow \operatorname{rep}\left(\operatorname{cl}\left(S_{w_{2 \delta(\mu d+1)+m}}\right)\right) \rightarrow \operatorname{rep}\left(S_{w_{2 \delta(\mu d+1)+m}}\right) \\
& \rightarrow \operatorname{rep}\left(\operatorname{cl}\left(S_{w_{3 \delta(\mu d+1)+m}}\right)\right) \rightarrow \operatorname{rep}\left(S_{w_{3 \delta(\mu d+1)+m}}\right) \\
& \vdots \quad \vdots \\
& \rightarrow \quad \operatorname{rep}\left(\operatorname{cl}\left(S_{w_{k}}\right)\right) \quad \rightarrow \quad \operatorname{rep}\left(S_{w_{k}}\right) \quad=\operatorname{rep}\left(S_{u}\right)
\end{aligned}
$$

It follows from Algorithm 2 that $\Pi$ is a path in $G$. We will show that the length of this path is at most $(2+\epsilon / 3)|p q|$.

We have shown already (see $(10)$ ) that the length of the first edge on $\Pi$ satisfies

$$
\left|p, \operatorname{rep}\left(\operatorname{cl}\left(S_{w_{0}}\right)\right)\right| \leq(1+4 / s) \ell_{0} .
$$


The length of the second edge satisfies

$$
\begin{aligned}
\left|\operatorname{rep}\left(\operatorname{cl}\left(S_{w_{0}}\right)\right), \operatorname{rep}\left(S_{w_{\delta(\mu d+1)+m}}\right)\right| & \leq\left|\operatorname{rep}\left(\operatorname{cl}\left(S_{w_{0}}\right)\right), p\right|+\left|p, \operatorname{rep}\left(S_{w_{\delta(\mu d+1)+m}}\right)\right| \\
& \leq(1+4 / s) \ell_{0}+\left|p, \operatorname{rep}\left(S_{w_{\delta(\mu d+1)+m}}\right)\right| .
\end{aligned}
$$

Since $p$ and $\operatorname{rep}\left(S_{w_{\delta(\mu d+1)+m}}\right)$ are both contained in $S_{u}$, it follows from Lemma 2.4 that

$$
\left|p, \operatorname{rep}\left(S_{w_{\delta(\mu d+1)+m}}\right)\right| \leq(2 / s)|p q| .
$$

Thus, the length of the second edge on $\Pi$ satisfies

$$
\left|\operatorname{rep}\left(\operatorname{cl}\left(S_{w_{0}}\right)\right), \operatorname{rep}\left(S_{w_{\delta(\mu d+1)+m}}\right)\right| \leq(1+4 / s) \ell_{0}+(2 / s)|p q| .
$$

Let $2 \leq j \leq m^{\prime}$. We have seen in (1) in the proof of Lemma 4.2 that the length of the edge

$$
\left(\operatorname{rep}\left(\operatorname{cl}\left(S_{w_{j \delta(\mu d+1)+m}}\right)\right), \operatorname{rep}\left(S_{w_{j \delta(\mu d+1)+m}}\right)\right)
$$

satisfies

$$
\left|\operatorname{rep}\left(\operatorname{cl}\left(S_{w_{j \delta(\mu d+1)+m}}\right)\right), \operatorname{rep}\left(S_{w_{j \delta(\mu d+1)+m}}\right)\right| \leq(1+4 / s) \ell_{j \delta(\mu d+1)+m} .
$$

Again, let $2 \leq j \leq m^{\prime}$. Since

$$
\operatorname{rep}\left(S_{w_{(j-1) \delta(\mu d+1)+m}}\right) \in S_{w_{j \delta(\mu d+1)+m}} \subseteq S_{x_{j \delta(\mu d+1)+m}}
$$

and

$$
\operatorname{rep}\left(\operatorname{cl}\left(S_{w_{j \delta(\mu d+1)+m}}\right)\right) \in S_{y_{j \delta(\mu d+1)+m}}
$$

it follows from Lemma 2.4 that the length of the edge

$$
\left(\operatorname{rep}\left(S_{w_{(j-1) \delta(\mu d+1)+m}}\right), \operatorname{rep}\left(\operatorname{cl}\left(S_{w_{j \delta(\mu d+1)+m}}\right)\right)\right)
$$

satisfies

$$
\left|\operatorname{rep}\left(S_{w_{(j-1) \delta(\mu d+1)+m}}\right), \operatorname{rep}\left(\operatorname{cl}\left(S_{w_{j \delta(\mu d+1)+m}}\right)\right)\right| \leq(1+4 / s) \ell_{j \delta(\mu d+1)+m} .
$$

We have shown that the length of $\Pi$ is at most

$$
(2 / s)|p q|+2(1+4 / s)\left(\ell_{0}+\sum_{j=2}^{m^{\prime}} \ell_{j \delta(\mu d+1)+m}\right) .
$$

The definition of $\ell_{0}, \ell_{1}, \ldots, \ell_{k}$ implies that this sequence is non-decreasing. Thus, $\ell_{0} \leq \ell_{\delta(\mu d+1)+m}$ and it follows that the length of $\Pi$ is at most

$$
(2 / s)|p q|+2(1+4 / s) \sum_{j=1}^{m^{\prime}} \ell_{j \delta(\mu d+1)+m} .
$$

Recall inequality (7) in the proof of Lemma 4.2, which states that

$$
\ell_{i} \leq \frac{1}{2} \ell_{i+\mu d+1}
$$


By applying this inequality $\delta$ times, we obtain

$$
\ell_{i} \leq\left(\frac{1}{2}\right)^{\delta} \ell_{i+\delta(\mu d+1)}
$$

For $i=j \delta(\mu d+1)+m$, this becomes

$$
\ell_{j \delta(\mu d+1)+m} \leq\left(\frac{1}{2}\right)^{\delta} \ell_{(j+1) \delta(\mu d+1)+m} .
$$

By repeatedly applying this inequality, we obtain, for $h \geq j$,

$$
\ell_{j \delta(\mu d+1)+m} \leq\left(\frac{1}{2}\right)^{(h-j) \delta} \ell_{h \delta(\mu d+1)+m} .
$$

For $h=m^{\prime}$, the latter inequality becomes

$$
\ell_{j \delta(\mu d+1)+m} \leq\left(\frac{1}{2}\right)^{\left(m^{\prime}-j\right) \delta} \ell_{k} .
$$

It follows that

$$
\begin{aligned}
\sum_{j=1}^{m^{\prime}} \ell_{j \delta(\mu d+1)+m} & \leq \sum_{j=1}^{m^{\prime}}\left(\frac{1}{2}\right)^{\left(m^{\prime}-j\right) \delta} \ell_{k} \\
& =\sum_{i=0}^{m^{\prime}-1}\left(\frac{1}{2}\right)^{i \delta} \ell_{k} \\
& \leq \sum_{i=0}^{\infty}\left(\frac{1}{2^{\delta}}\right)^{i} \ell_{k} \\
& =\frac{2^{\delta}}{2^{\delta}-1} \ell_{k}
\end{aligned}
$$

According to 2 in the proof of Lemma 4.2, we have

$$
\ell_{k} \leq(1+4 / s)|p q| \text {. }
$$

We have shown that the length of the path $\Pi$ is at most

$$
\left(2 / s+2(1+4 / s)^{2} \frac{2^{\delta}}{2^{\delta}-1}\right)|p q|
$$

Our choices of $s$ and $\delta$ (see Algorithm 2) imply that $2 / s \leq \epsilon / 6,(1+4 / s)^{2} \leq 1+\epsilon / 36$ and $\frac{2^{\delta}}{2^{\delta}-1} \leq 1+\epsilon / 36$. Therefore, the length of $\Pi$ is at most

$$
\left(\epsilon / 6+2(1+\epsilon / 36)^{2}\right)|p q| \leq(2+\epsilon / 3)|p q|,
$$

where the latter inequality follows from our assumption that $0<\epsilon<1$. This completes the proof. 
Lemma 5.3 Let $n=|S|$. The graph $G$ computed by Algorithm 2 is a $(5+\epsilon)$-spanner of the complete $k$-partite graph $K_{C_{1} \ldots C_{k}}$ and the number of edges of this graph is $O(n)$. The running time of Algorithm 2 is $O(n \log n)$.

Proof: The proof for the upper bound on the stretch factor is similar to the one of Lemma 4.3 . The difference is that instead of the value $t^{\prime}$ that was used in the proof of Lemma 4.3 , we now use the value $t^{\prime}=2+\epsilon / 3$ of Lemma 5.2 . Thus, the stretch factor for the base case of the induction and for Case 1 is at most

$$
(1+4 / s)+2 t^{\prime}=5+4 / s+2 \epsilon / 3,
$$

which is at most $5+\epsilon$, because of our choice for $s$ in Algorithm 2. For Cases 2 and 3, the stretch factor is at most (see (8) in the proof of Lemma 4.3 , where $t=5+\epsilon$ )

$$
t^{\prime}+(1+4 / s)+4 t / s=3+\epsilon / 3+(4 / s)(6+\epsilon),
$$

which is at most $5+\epsilon$, again because of our choice for $s$. Finally, the stretch factor for Case 4 is at most (see $(9)$ in the proof of Lemma 4.3 , where $t=5+\epsilon$ )

$$
(1+4 / s)+8 t / s=1+(4 / s)(11+2 \epsilon),
$$

which is at most $5+\epsilon$, because of our choice for $s$.

The analysis for the number of edges is the same as in Lemma 4.1, except that the number of edges that are added to each $c$-node in the modified for-loop is $2 \delta(\mu d+1)$ instead of one as is in Algorithm 1. Finally, the analysis of the running time is the same as in Lemma 4.4.

We have proved the following result.

Theorem 5.4 Let $k \geq 2$ be an integer, let $S$ be a set of $n$ points in $\mathbb{R}^{d}$ which is partitioned into $k$ subsets $C_{1}, C_{2}, \ldots, C_{k}$, and let $0<\epsilon<1$ be a real constant. In $O(n \log n)$ time, we can compute a $(5+\epsilon)$-spanner of the complete $k$-partite graph $K_{C_{1} \ldots C_{k}}$ having $O(n)$ edges.

\section{Improving the Stretch Factor}

We have shown how to compute a $(5+\epsilon)$-spanner with $O(n)$ edges of any complete $k$-partite graph. In this section, we show that if we are willing to use $O(n \log n)$ edges, the stretch factor can be reduced to $3+\epsilon$. We start by showing that a stretch factor less than 3 , while using $O(n \log n)$ edges, is not possible.

Theorem 6.1 Let $c>0$ be a constant and let $n$ and $k$ be positive integers with $2 \leq k \leq n-$ $2 c \sqrt{n \log n}$. For every real number $0<\epsilon<1$, there exists a complete $k$-partite geometric graph $K$ with $n$ vertices such that the following is true: If $G$ is any subgraph of $K$ with at most $c^{2} n \log n$ edges, then the stretch factor of $G$ is at least $3-\epsilon$.

Proof: Let $D_{1}, D_{2}$, and $D_{3}$ be three disks of radius $\epsilon / 12$ centered at the points $(0,0),(1+\epsilon / 6,0)$, and $(2+\epsilon / 3,0)$, respectively. We place $(n-k+1) / 2$ red points inside $D_{1}$ and $(n-k+1) / 2$ blue points inside $D_{2}$. The remaining $k-2$ points are placed inside $D_{3}$ and each of these points has a 
distinct color (which is not red or blue). Let $K$ be the complete $k$-partite geometric graph defined by these $n$ points. We claim that $K$ satisfies the claim in the theorem.

Let $G$ be an arbitrary subgraph of $K$ and assume that $G$ contains at most $c^{2} n \log n$ edges. We will show that the stretch factor of $G$ is at least $3-\epsilon$.

Assume that $G$ contains all red-blue edges. Then the number of edges in $G$ is at least $((n-k+2) / 2)^{2}$. Since $k \leq n-2 c \sqrt{n \log n}$, this quantity is larger than $c^{2} n \log n$. Thus, there is a red point $r$ and a blue point $b$, such that $(r, b)$ is not an edge in $G$. The length of a shortest path in $G$ between $r$ and $b$ is at least 3. Since $|r b| \leq 1+\epsilon / 3$, it follows that the stretch factor of $G$ is at least $\frac{3}{1+\epsilon / 3}$, which is at least $3-\epsilon$.

Theorem 6.2 Let $k \geq 2$ be an integer, let $S$ be a set of $n$ points in $\mathbb{R}^{d}$ which is partitioned into $k$ subsets $C_{1}, C_{2}, \ldots, C_{k}$, and let $0<\epsilon<1$ be a real constant. In $O(n \log n)$ time, we can compute a $(3+\epsilon)$-spanner of the complete $k$-partite graph $K_{C_{1} \ldots C_{k}}$ having $O(n \log n)$ edges.

Proof: Consider the following variant of the WSPD. For every pair $\{X, Y\}$ in the standard WSPD, where $|X| \leq|Y|$, we replace this pair by the $|X|$ pairs $\{\{x\}, B\}$, where $x$ ranges over all points of $X$. Thus, in this new WSPD, each pair contains at least one singleton set. Callahan and Kosaraju [4] showed that this new WSPD consists of $O(n \log n)$ pairs.

We run Algorithm 2 on the set $S$, using this new WSPD. Let $G$ be the graph that is computed by this algorithm. Observe that Lemma 5.2 still holds for $G$. In the proof of Lemma 5.3 of the upper bound on the stretch factor of $G$, we have to apply Lemma 5.2 only once. Therefore, the stretch factor of $G$ is at most $3+\epsilon$.

\section{Conclusion}

We have shown that for every complete $k$-partite geometric graph $K$ in $\mathbb{R}^{d}$ with $n$ vertices and for every constant $\epsilon>0$,

1. a $(5+\epsilon)$-spanner of $K$ having $O(n)$ edges can be computed in $O(n \log n)$ time,

2. a $(3+\epsilon)$-spanner of $K$ having $O(n \log n)$ edges can be computed in $O(n \log n)$ time.

The latter result is optimal for $2 \leq k \leq n-\Theta(\sqrt{n \log n})$, because a spanner of $K$ having stretch factor less than 3 and having $O(n \log n)$ edges does not exist for all complete $k$-partite geometric graphs.

We leave open the problem of determining the best stretch factor that can be obtained by using $O(n)$ edges.

Future work may include verifying other properties that are known for the general geometric spanner problem. For example, is there a spanner of a complete $k$-partite geometric graph that has bounded degree? Is there a spanner of a complete $k$-partite geometric graph that is planar? From a more general point of view, it seems that little is known about geometric spanners of graphs other than the complete graph. The unit disk graph received great attention, but there are a large family of other graphs that also deserve attention. 


\section{References}

[1] I. Althöfer, G. Das, D. P. Dobkin, D. Joseph, and J. Soares. On sparse spanners of weighted graphs. Discrete \& Computational Geometry, 9:81-100, 1993.

[2] Prosenjit Bose, Paz Carmi, Mathieu Couture, Anil Maheshwari, Michiel Smid, and Norbert Zeh. Geometric spanners with small chromatic number. In Proceedings of the 5th Workshop on Approximation and Online Algorithms, Lecture Notes in Computer Science, Berlin, 2007. Springer-Verlag.

[3] P. B. Callahan and S. R. Kosaraju. Faster algorithms for some geometric graph problems in higher dimensions. In Proceedings of the 4th ACM-SIAM Symposium on Discrete Algorithms, pages 291-300, 1993.

[4] Paul B. Callahan and S. Rao Kosaraju. A decomposition of multidimensional point sets with applications to $k$-nearest-neighbors and $n$-body potential fields. J. ACM, 42(1):67-90, 1995.

[5] J. Gudmundsson and M. Smid. On spanners of geometric graphs. In Proceedings of the 10th Scandinavian Workshop on Algorithm Theory, volume 4059 of Lecture Notes in Computer Science, pages 388-399, Berlin, 2006. Springer-Verlag.

[6] Giri Narasimhan and Michiel Smid. Geometric Spanner Networks. Cambridge University Press, New York, NY, USA, 2007.

[7] Bhaskaran Raman and Kameswari Chebrolu. Design and evaluation of a new MAC protocol for long-distance 802.11 mesh networks. In MobiCom '05: Proceedings of the 11th annual international conference on Mobile computing and networking, pages 156-169, New York, NY, USA, 2005. ACM Press.

[8] J. S. Salowe. Constructing multidimensional spanner graphs. International Journal of Computational Geometry \& Applications, 1:99-107, 1991.

[9] P. M. Vaidya. A sparse graph almost as good as the complete graph on points in $K$ dimensions. Discrete \& Computational Geometry, 6:369-381, 1991. 